\title{
Design and Application of Electrical Equipment Overheating Monitoring Based on Wireless Sensor
}

\author{
https://doi.org/10.3991/ijoe.v13i07.7277 \\ Pinghui $\mathrm{Hu}$ \\ Hunan International Economics University, Changsha, China \\ Honghua Liu ${ }^{(\varpi)}$ \\ Hunan International Economics University, Changsha, China \\ Lhh1127@126.com
}

\begin{abstract}
A new scheme is proposed to solve the practical problems in the intelligent monitoring of $220 \mathrm{kV}$ substation equipment. By analyzing the changes in temperature of substation electrical equipment, it applies wireless sensor network technology in the inverter station based on the running condition of the equipment. In addition, the acquisition, processing, and communication of temperature information is used as the major research object, and a kind of intelligent monitoring of substation equipment system based on wireless sensor network is designed. By using C805IF996 chip and nRF905 chip as the main chips, the hardware and software design of the temperature data acquisition node and sink node are realized. The result show that the electrical equipment based on wireless sensor has a great impact in overheating monitoring. Based on the above finding, a conclusion is drawn that wireless sensor provides an effective solution for the application of intelligent monitoring of electrical equipment.
\end{abstract}

Keywords-wireless sensor, temperature monitoring, acquisition node, sink node

\section{Introduction}

For high voltage electrical equipment in substation, it is easy to appear insulation aging or too large contacted resistance phenomenon during the long time of operation, and it will lead to overheating, cause fires and large scale blackouts, and bring about significant economic losses. Since that a large proportion of the temperature change of substation high voltage electrical equipment can reflect the running state of the equipment, it is of practical value for make a real-time temperature monitoring on the substation equipment [1]. Along with the continuous development of computer technology, wireless communication and sensor technology, wireless sensor network (Wireless Sensor Network, WSN) with the integration of sensor technology, information processing technology and wireless communication technology comes into being. This paper applies the wireless sensor network technology in the inverter sta- 
tion, takes the acquisition, processing, and communication of temperature information as the major research objects, and designs a kind of substation equipment intelligent monitoring system based on wireless sensor network.

In the process of system design, the hardware and software design of the temperature data acquisition node and sink node are realized by using C805IF996 chip and nRF905 chip as the main chips. The temperature data acquisition node is composed of a temperature acquisition module, a microprocessor module, a wireless communication module and a power module; the sink node consist of a wireless communication module, a microprocessor module, a serial communication module, and a power module. Take Time Division Multiple Access as the communication protocol between the temperature data acquisition node and the sink node. The temperature data acquisition nodes are distributed throughout the substation equipment temperature data, and then wirelessly sent to the sink node; the sink node analyzes and integrates the temperature data collected, then transfer to the 485 bust wait for the bus monitoring computer response [2]. Through the hardware circuit of the temperature data acquisition node and sink node, and the test of mutual communication between them, it has achieved the expected goals and proved the rationality of the design, which provides an effective solution for the application of intelligent monitoring of electrical equipment.

\section{Overview}

The temperature monitoring system of substation equipment based on wireless sensor network is divided into three layers in general: temperature data acquisition layer, communication layer and management layer.

The temperature data acquisition layer is at the bottom of the system. It is composed of multiple temperature data acquisition node and sink node deployed in the substation environment, which is used for the temperature of important substation equipment, like collecting bus connector, outdoor knife switch, transformer winding, various switch-gears and so on. The temperature data collection points are placed in the vicinity of the above equipment, the temperature data of high voltage electrical equipment collected is conducted with analysis and processing, and then wirelessly transmitted to the sink node. The sink node gathers and processes the data, and then waits for the instructions of monitoring computer, and does well the preparation and monitoring of computer communication.

Communication layer refers to the communication between the sink node and the monitoring computer, which uses the RS-485 bus protocol, the middle uses 485 to 232 module to achieve the conversion from 485 electrical level to 232 electrical level, and the monitoring computer communicates with a plurality of sink node through the 485 bus [3]. They will use a master-slave response method to realize communication, different addresses between each sink node are the unique identifier provided for monitoring computer accessing to the sink node.

Management layer indicates the process of monitoring computer analyzing and processing the temperature data of substation equipment collected by the computer. 
The monitoring computer can, according to the needs of the substation staffs, collect the temperature information installed nearby each substation equipment, and complete the temperature data processing, parameter setting, storage, real-time display, temperature alarm and other functions.

\section{$3 \quad$ Materials and methods}

\subsection{Hardware design of monitoring system for substation equipment}

The hardware architecture of the system is mainly composed of three parts, including the hardware part of the temperature data acquisition node, the hardware part of the sink node and the monitoring computer.

Hardware design of temperature data acquisition node: The main function of the temperature data acquisition node is to acquire the temperature parameter of substation relevant electrical equipment, to process the temperature, and to wirelessly transmit the temperature data. According to the functional requirements of temperature data acquisition nodes, in the design of hardware, put it into the temperature data acquisition module, the processor module, the wireless communication module, and the power supply module. The temperature data acquisition module mainly consists of DS18B20 temperature sensor, which is used to acquire the temperature information of the monitoring equipment, and transmit to the CPU core control module. The processor module is mainly composed of C8051F996 microprocessor and its peripheral circuits, which is the core control module of the system. CPU uses Silicon Labs 8051 microprocessor C8051F996 to perform processing, communication and other functions of data [4]. The wireless communication module is mainly composed of nRF905 chip and its peripheral circuits, which is the RF communication module of the node. The chip is the RF $433 / 868 / 915 \mathrm{MHz}$ chip produced by NORDIC company, used for wireless transmission of data packets information. The overall structure of the block diagram of temperature data acquisition node is shown in figure 1.

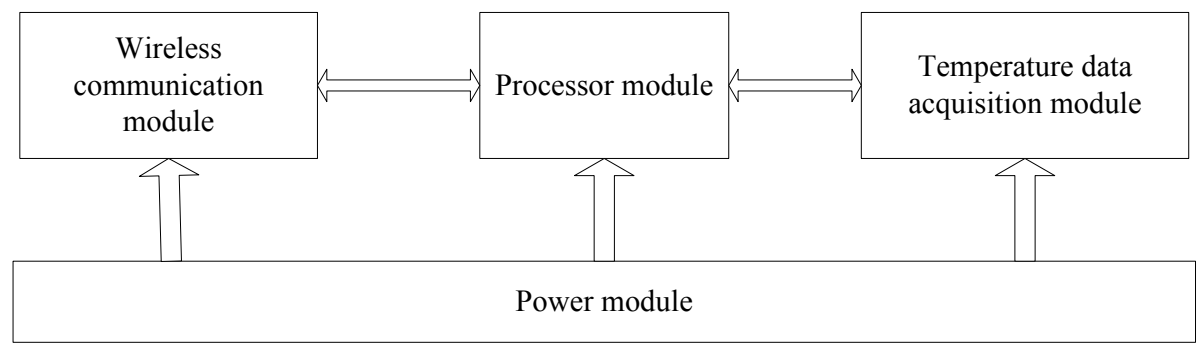

Fig. 1. The overall structure of the block diagram of temperature data acquisition node

Temperature acquisition module: In order to meet the requirements of substation equipment monitoring, the design of temperature data acquisition uses the DS18B20 temperature sensor launched by Dallas company. The DS18B20 temperature sensor is a temperature sensor that supports single bus interface, and all of the sensing elements 
and the conversion circuit are integrated in the internal circuit of a transistor. Single bus uses a single signal line for data transmission, and conducts bidirectional transmission of data, which has the advantages of saving I/O mouth line resources, simple in structure, low in cost, convenient for bus expansion and maintenance and so on [5]. Compared with other kinds of temperature sensors, DS18B20 has a unique single bus interface mode. When connected with the microprocessor, it requires only one port line, then it can realize bidirectional communication between microprocessor and DS18B20; applicable voltage range is $3.0 \sim 5.5 \mathrm{~V}$, powered by a data line in the power source parasitic mode; DS18B20 temperature range: -55 to +125 DEG C, the accuracy in -10 to +85 DEG $C$ is \pm 0.5 DEG $C$.

Processor module: The processor module is the core part of the temperature acquisition node. It first of all achieves the acquisition of the environmental temperature data through the temperature acquisition unit circuit, and then processes the temperature data collected and sends to the sink node by the wireless communication module. Therefore, the interface circuit between the microprocessor chip C8051F996 and the temperature acquisition module DS18B20 and the wireless communication module nRF905 RF chip is the main component of the microprocessor unit circuit.

Wireless communication module: nRF905 is the chip used by the wireless communication module in the temperature acquisition node. The wireless communication module circuit includes the interface circuit with C8051F996, crystal oscillator circuit and antenna matching circuit.

Hardware circuit design of sink node: The sink node is the core part of the whole monitoring system. First of all, it is mainly responsible for the temperature data collected by the temperature acquisition points by means of wireless communication; secondly, it is mainly responsible for the analysis and integration of the temperature signal collected, transmitted to the 485 bus and waiting for a response from the monitoring computer.

The sink node comprises a power supply module, a wireless communication module, a processor module, and a communication module. The overall structure of the hardware circuit is shown in figure 2. The power supply module uses two kinds of power chips, LM2578 and AS1117, respectively. The processor module uses C8051F996 chip to control and manage the sink node. The wireless communication module uses the nRF905 radio frequency chip to undertake the wireless communication with the temperature data acquisition nodes. The serial communication module uses the SP485R chip to undertake the data communication with the substation monitoring computer. The chips used in the wireless communication module and the processor module that used in the temperature data acquisition node are the same.

Power module: The power supply module is the energy supply part of the sink node. According to the needs of the different units in the sink node, the power module needs to provide the voltage of $5 \mathrm{~V}$ and $3.3 \mathrm{~V}$. $3.3 \mathrm{~V}$ is to provide voltage for the C8051F996 chip and nRF905 RF chip. Therefore, in the design of power modules, make use of LM2576 and AS1117 two power chips, respectively.

Serial communication module: The serial communication module is mainly responsible for the communication between the host computer of substation and the sink node. It transfers the temperature data processed by the sink node to the host 
computer, and then waits for the computer to respond. In order to make the nodes have a long communication distance, it is necessary to enhance the ability of the system to resist external interference, and increase the number of aggregation nodes on the bus port [6]. As a result, we make use of the RS-485 serial communication standard interface.

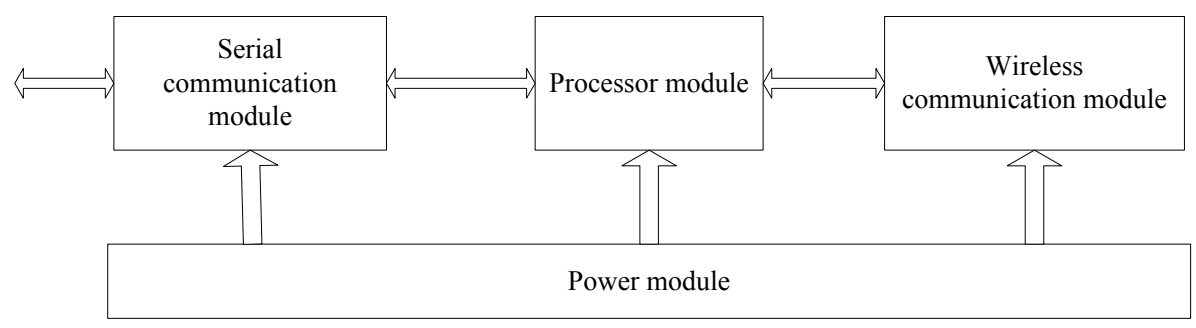

Fig. 2. The overall structure of the sink node circuit

\subsection{Software design of monitoring system for substation equipment}

According to the overall framework and the specific functions to achieve of the substation equipment temperature monitoring system, the software design of monitoring system should include the temperature data acquisition node software, sink node software, and computer monitoring software. In the process of system software design, divide the whole system software into several parts. First of all, singly compile the program for the single module, and make a separate test debugging, so as to achieve the functions that a single module should have, and finally make an integrated debugging to achieve the whole function of the system. A temperature data acquisition node software includes the following three parts: the temperature acquisition module, the whole node scheduling module, and the wireless communication module [7]; the sink node software includes the following three parts: the wireless communication module, the whole node scheduling module, and the serial communication module; the monitoring computer software includes the design of man-machine interface, communication function interface and data processing module. The overall frame of system software is shown in figure 3.

Temperature data acquisition node software design: The temperature data acquisition node mainly completes the collection and processing of the temperature data, and transmits the collected temperature data to the sink node in time. The temperature data acquisition node is completed in one-time frame cycle in turn: receiving the control command sent by the sink node, collecting the temperature data, sending the temperature data in time delay, and entering the dormant state. Figure 4 shows the software flow chart of the temperature data acquisition node.

The temperature data acquisition module mainly realizes the communication between the microprocessor C8051F996 and the DS18B20 temperature sensor. The temperature acquisition module in the design uses DS18B20 as the temperature acquisition chip, and according to the characteristics of the DS18B20, makes use of external power supply access for its power supply. 


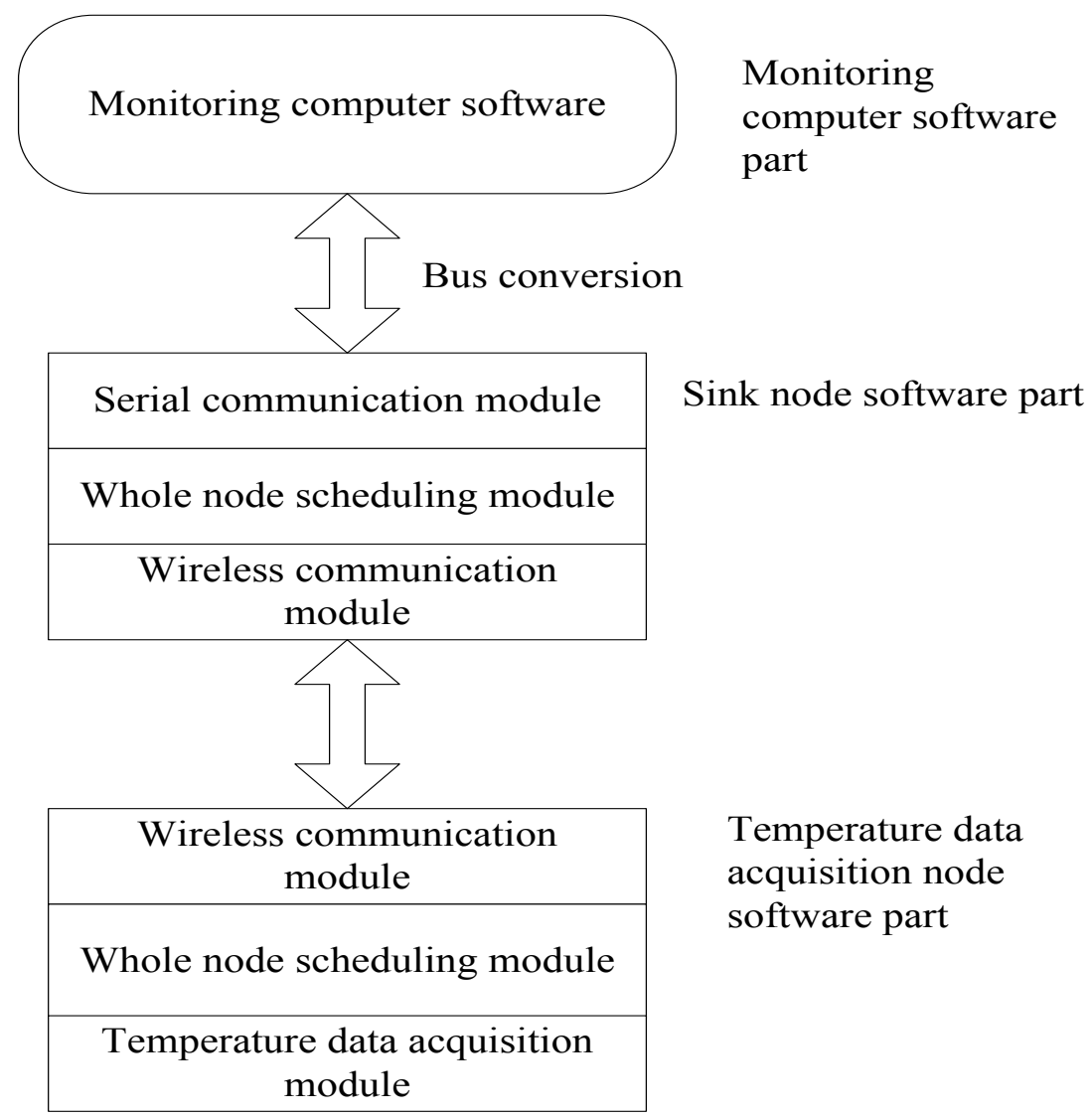

Fig. 3. The overall framework of system software

Sink node software design: The sink node is the core part of high voltage electrical equipment in substation temperature monitoring. On the one hand, it is mainly responsible for the communication between the temperature data acquisition nodes and the temperature data collected by data acquisition node; on the other hand, it is mainly responsible for the processing of temperature data collected, transmitted to the bus and waiting for the communication between PC [8]. The sink node needs to complete the following steps in one frame period: to send synchronization command to the temperature data acquisition node, waiting for the temperature data acquisition node to return data; to receive the data sent by the temperature data acquisition node, and to communicate with substation monitoring computer. As shown in Figure 5, it is the flow chart of the main program software of the sink node. 


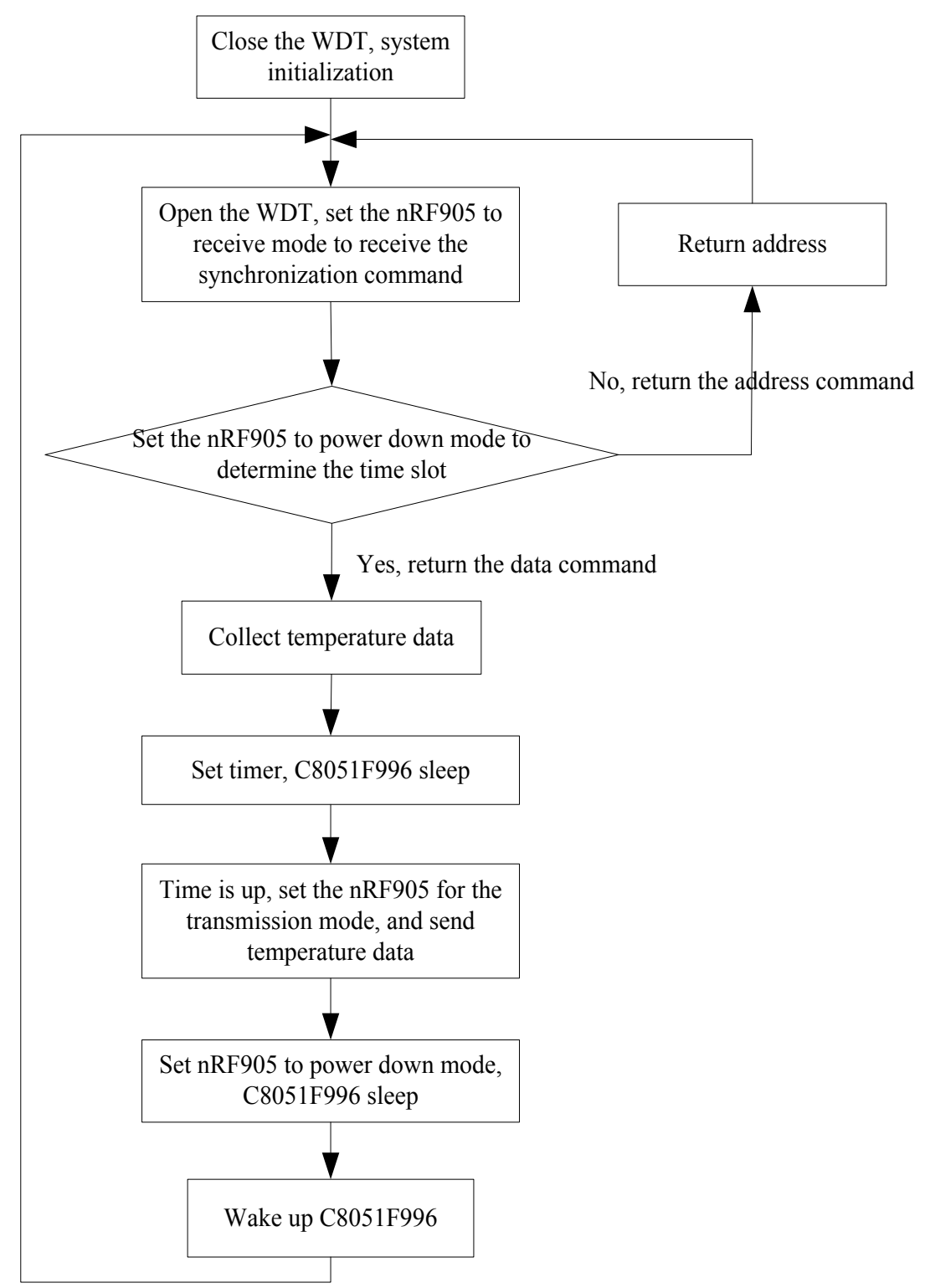

Fig. 4. The software flow chart of the temperature data acquisition node 


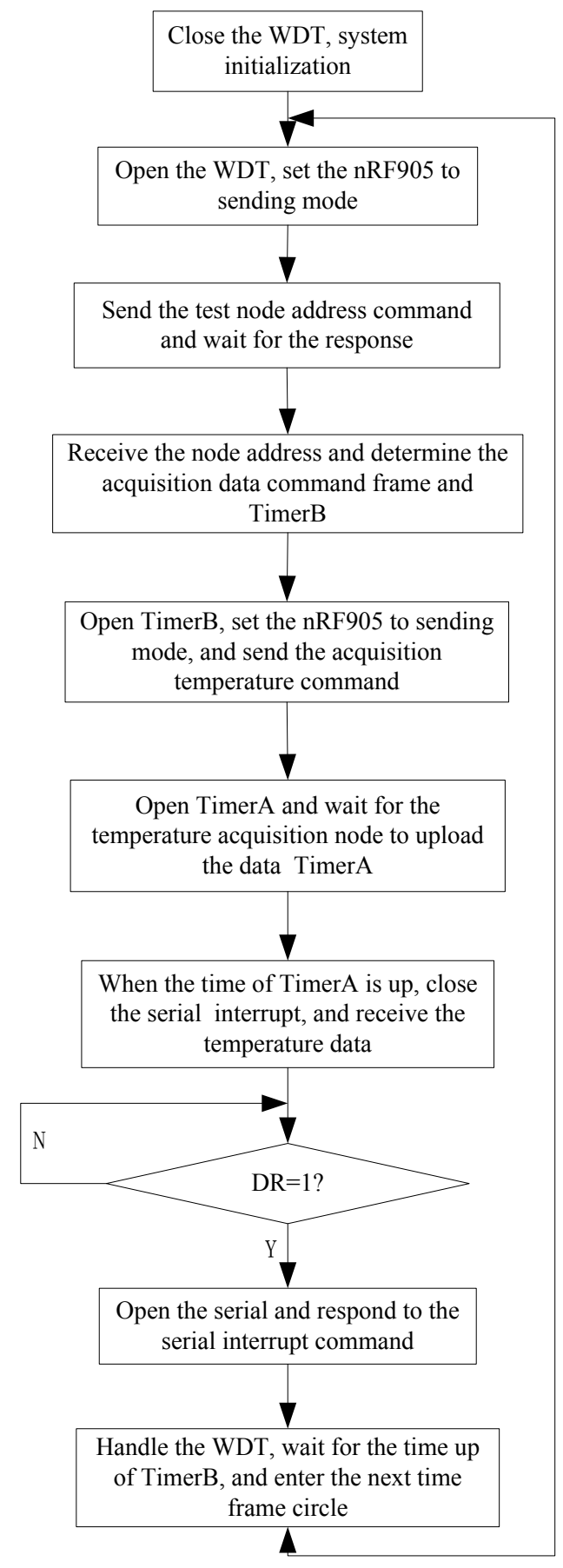

Fig. 5. The sink node software flow chart 


\section{$4 \quad$ Results}

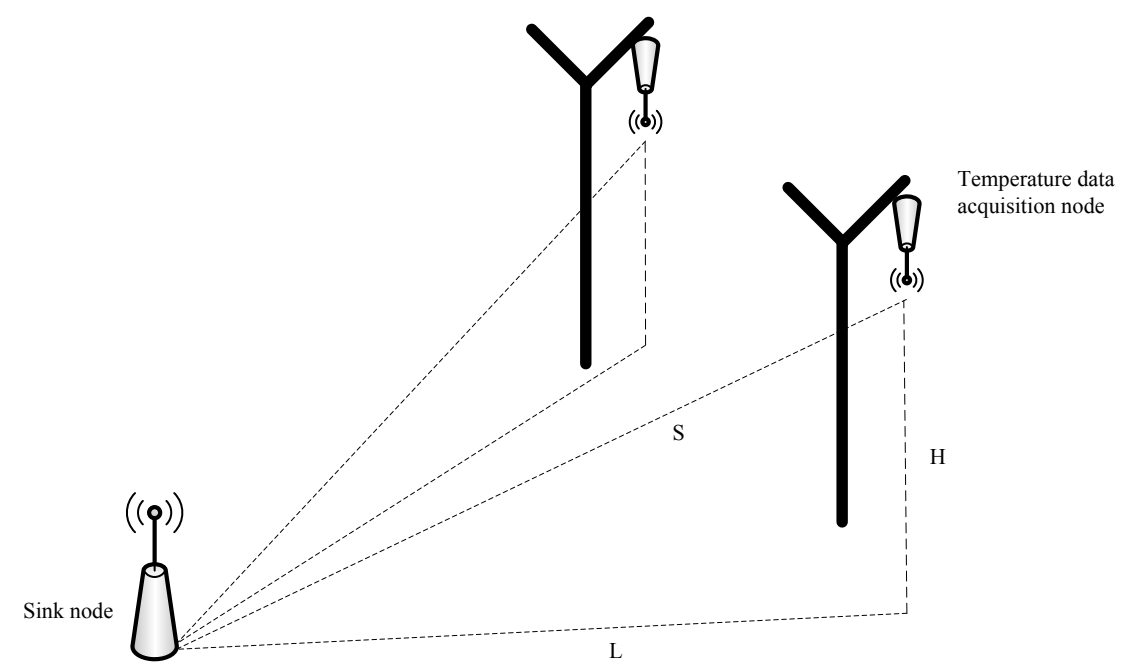

Fig. 6. Test of communication between temperature data acquisition node and sink node

As shown in figure 6, the temperature data acquisition node is connected to the isolation switch, and the sink node placed somewhere on the ground, able to measure the height from the temperature data acquisition node placed at the isolation switch to the ground is $\mathrm{H}$, and the vertical projection of the sink node distance to the temperature data acquisition node in the ground is L. Then, according to the Pythagorean theorem, we can calculate the distance from the sink node to the temperature data acquisition nodes.

In order to verify the reliability of communication between the temperature data acquisition node and the sink node, we make two temperature data acquisition nodes and a sink node, and observe whether the communication between the two temperature data acquisition nodes and sink node is successful through the serial debugging software. First of all, connect the sink node with the computer, and then power on the two temperature data acquisition nodes, open a serial debugging software, and select the COM3 port, the baud rate is 19200 .

The above data is the data of the two temperature data acquisition node collected directly by the sink node, the test uses two data acquisition nodes. "00 02" and "00 04" refer to the temperature data acquisition node address, "01 61" and "01 4D" shown after the address are the temperature data collected by two nodes. These two nodes are tested in the occluded and open space, able to carry out normal communication in $150 \mathrm{~m}$. Through the DS18B20 temperature conversion formula, we can calculate the temperature of the two temperature data acquisition nodes: 22.0625 and 20.8125 , and compared with the actual situation, the data acquisition is basically correct. The above test shows that the temperature data acquisition node and the sink node can communicate normally, collect and transfer the temperature data. 
Paper-Design and Application of Electrical Equipment Overheating Monitoring Based on Wireless...

\section{Conclusion}

Through the test on the temperature dada acquisition node, the validity of temperature monitoring system based on wireless sensor network is proved. And the reliability of communication between the temperature data acquisition node and the sink node is verified. The experiment result proves that the content of the substation equipment monitoring is extensive and complex, and the $220 \mathrm{kV}$ wireless monitoring system of the substation equipment temperature is only a large set of substation electrical equipment monitoring system. WSN can be widely used in the monitoring of high voltage electrical equipment in substation. In addition, it is remained to be studied for more sensor nodes for collecting substation and high voltage electrical equipment related to environmental information. Based on the result data, we can concluded that wireless sensor network combines the advantages of sensor technology, embedded computer technology, modern network technology, wireless communication technology and distributed intelligent information processing technology, which is an excellent choice for monitoring the electrical problem of overheating. The multi hop function and topology flexibility of wireless sensor network can effectively avoid the attenuation of wireless signals by various buildings and devices. The self-organization and adaptability of the network can greatly improve the reliability of the on-line monitoring system.

\section{References}

[1] Alhusban, A. A., Breadmore, M. C., Gueven, N., \& Guijt, R. M. (2016). Capillary electrophoresis for automated on-line monitoring of suspension cultures: Correlating cell density, nutrients and metabolites in near real-time. Analytica chimica acta, 920: 94-101. https://doi.org/10.1016/j.aca.2016.03.034

[2] Genner, A., Gasser, C., Moser, H., Ofner, J., Schreiber, J., \& Lendl, B. (2017). On-line monitoring of methanol and methyl formate in the exhaust gas of an industrial formaldehyde production plant by a mid-IR gas sensor based on tunable Fabry-Pérot filter technology. Analytical and bioanalytical chemistry, 409(3): 753-761. https://doi.org/10.1007/s002 16-016-0040-9

[3] Kim, H., Park, H., \& Yoe, H. (2015). Design and implementation of cowshed methane gas monitoring system based on wireless sensor network. ASIA LIFE SCIENCES, 11: 31-42.

[4] Sharma, M. K., Wieringa, F. P., Frijns, A. J., \& Kooman, J. P. (2016). On-line monitoring of electrolytes in hemodialysis: on the road towards individualizing treatment. Expert Review of Medical Devices, 13(10): 933-943. https://doi.org/10.1080/17434440.2016.123049 4

[5] X' ingkui, M., Qisheng, H., Yudi, X., Shifa, L., Zhe, Z., \& Andersen, M. A. (2016, October). Wireless power supply via coupled magnetic resonance for on-line monitoring wireless sensor of high-voltage electrical equipment. In Industrial Electronics Society, IECON 2016-42nd Annual Conference of the IEEE (pp. 4582-4587). IEEE.

[6] Yu, H., Qian, Z., Yao, J., \& Xia, J. (2016, January). Research of on-line monitoring equipment for power capacitor based on wireless sensor network. In Seventh International Symposium on Precision Mechanical Measurements(pp. 99030V-99030V). International Society for Optics and Photonics. https://doi.org/10.1117/12.2211347 
Paper-Design and Application of Electrical Equipment Overheating Monitoring Based on Wireless...

[7] Yu, Z. Z., Wei, C. G., Li, Z. G., Yang, S., Du, Y., \& Li, X. F. (2014). Based on Wireless Sensor Technology for Substation Equipment Temperature Monitoring System. In Advanced Materials Research (Vol. 986, pp. 1704-1708). Trans Tech Publications. https://doi.org/10.4028/www.scientific.net/amr.986-987.1704

[8] Zhou, K., Xiong, Q., He, M., Dong, L., \& Chen, Y. (2016). An On-line Monitoring System for Over-voltages Based on a Two-stage Voltage Divider and Field Measurement Results in Medium-voltage Grids. Electric Power Components and Systems, 44(12): 1345-1356. https://doi.org/10.1080/15325008.2015.1028117

\section{$7 \quad$ Authors}

Pinghui Hu is with Hunan International Economics University, Changsha, China (hupinghui2008@126.com).

Honghua Liu (corresponding author) is with Hunan International Economics University, Changsha, China (Lhh1127@126.com).

Article submitted 10 June 2017. Published as resubmitted by the authors 14 July 2017. 